\title{
Designing and Investigating New Ways of Interactive Whiteboard Use in Physics Instruction
}

\author{
Bor Gregorcic*, Eugenia Etkina† and Gorazd Planinsic* \\ *University of Ljubljana, Faculty for mathematics and physics, Jadranska 19, Ljubljana, Slovenia \\ $\dagger$ Graduate school of education, Rutgers University, 10 Seminary Place, New Brunswick, NJ 08901-1183
}

\begin{abstract}
Researchers on the topic of Interactive Whiteboard (IWB) use in education stress that while important, teachers' mastering of the technological and interactive aspects of the IWB is not enough for its productive use. In order to get the most out of the relatively expensive investment in IWBs, much of the attention has to be given to the student active engagement during the lessons. In a qualitative study, we investigated how students interacted with an IWB during a lesson that was designed based on the principles of guided-inquiry and kinesthetic engagement of learners. Our study used activity theory as a descriptive framework for analysis. The topic of the lessons was Kepler's laws. We have found that students are well adapted to a shift from classical board-like use of the IWB that is predominant in a high school setting to touchscreen-like use, where kinesthetic engagement and creative graphical input play a central role. Students engaged in collaborative sense-making, discovering patterns, setting and testing hypotheses with little intervention by the researcher who was present in class during small group activities. However, we found that appropriate scaffolding by the researcher was very important as it allowed the students to avoid pitfalls of technology use. Such scaffolding requires that the person guiding student work with the IWBs possesses technological, pedagogical, and content knowledge.
\end{abstract}

Keywords: IWB, activity theory, Kepler's laws, computer-supported collaborative learning, Algodoo, kinesthetic, gesture PACS: 01

\section{INTRODUCTION}

The interactive whiteboard (IWB) has become a fairly common piece of instructional equipment in the western world. As technology prices come down, IWBs will likely become more widely available. However, using the IWB as a traditional writing and drawing tool or as a computer-projector setup does not justify the relatively expensive investment into the touch-sensitive surface of the IWB.

Previous research suggests that the benefits of IWB are connected to the pedagogy surrounding its use [1]. The so-called technical interactivity must therefore be supported by appropriate pedagogical approaches [2]. Teachers or instructional designers must possess a complex interplay of technological, pedagogical and content knowledge also referred to as TPACK [3] to get the most out of IWBs.

The goal of our qualitative study is explore what types of activities leverage affordances that are specific to IWB. To investigate this, we have designed an activity that utilizes the IWB combined with Algodoo software (www.algodoo.com). Algodoo is a 2-D physics sandbox software, enabling users to create and manipulate customized objects in a virtual environment governed by certain physical laws. In designing the activity described and analyzed in this paper, we have focused primarily on taking advantage of the kinesthetic and socially interactive affordances of the IWB and accompanying software. The design is built on principles derived from embodied cognition [4,5], which puts forward the importance of kinesthetic engagement of learners and Vygotskyan social psychology [6], which regards social interaction a central mechanism of learning. A field of educational study called computer-supported collaborative learning [7] stresses the importance of qualitative studies addressing concrete examples of student collaborative learning with the support of technology.

We want to make it clear that our study, being a case study, follows a qualitative research paradigm and does not aim to produce widely generalizable results [8]. Instead, by observing student-centered IWB activity and describing it using a theoretical framework, we try to identify student behaviors that are seen to be beneficial for learning and describe the circumstances that surrounded these behaviors.

In the activity on the topic of orbital motion of planets, students were asked to explore the influence of a massive object on nearby smaller objects in a virtual environment using the IWB. They could conduct the exploration by drawing and throwing objects in the gravitational field of the central object. The main goal of the activity for the students was to discover the basic principles of orbital motion - Kepler's laws. The topic was selected because of a lack of real experiments that 
would allow students to engage in inquiry-based activities and because the Algodoo lends itself to a meaningful student kinesthetic and graphical input. In our study, we have observed 2 groups of 3 students engaging in the activity described above.

Research questions that we attempt to answer in this study are: What will the learners do if they are given full control of the IWB? Will the designed activity encourage social interactions and collaborative learning? To what extent will the students be kinesthetically engaged?

\section{ACTIVITY THEORY}

In order to analyze the processes that occurred while students were working through the problem, we have chosen to use a descriptive framework called activity theory (AT) that has its origins in the social psychology of Lev Vygotsky. It was developed by his student Alexei Leontiev and Yrjo Engestrom [9,10]. AT builds on Vygotsky's ideas of transformations along the external/internal dimension of cognition, through the processes of externalization (representing mental processes via external means) and internalization (an important mechanism of learning in social settings). AT views cognition as a process distributed between the individual and his/her surroundings, including other people.

In an activity, the subject acts on the object of interest to reach his or her goal. While doing this, the subject uses tools that can be physical (computers, writing tools, etc.) or mental (memorization techniques etc.). The use of tools is culturally mediated and negotiated among the members of the communities who use them.

The subjects relate to the surrounding community through formal and informal rules (such as rules guiding classroom activities). The community divides the tasks through division of labor. To gain an insight into an activity system, one has to take into account all its components (Fig. 1) and their interconnectedness. AT is therefore a holistic framework for qualitative analysis of human activity.

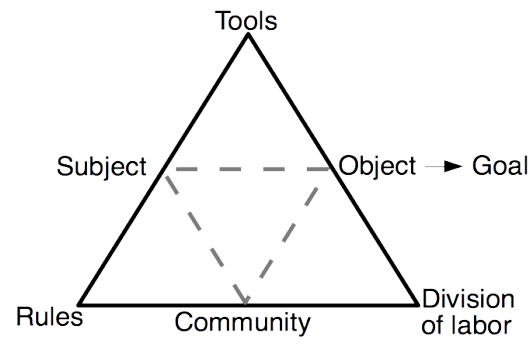

FIGURE 1. The structure of the activity system suggested by Engestrom [10]. The interactions among the subject community and the object are mediated by tools, rules and the division of labor.
AT is most often used in, but not limited to, qualitative studies of institutionalized human behavior and in the design of human-computer interaction [8,9].

AT breaks the activity down to three levels [9]. The top level is called activity and it refers to combined efforts towards reaching the main goal. In our case this would mean achieving learning. The second level is called actions. Actions are efforts that lead to achievement of goals. An example of an action in our case would be sending a planet into an eccentric orbit around the Sun. Operations are the lowest level of activity. They differ from actions as they are performed automatically. An example would be selecting the desired object on the screen and moving it to a different location. Actions can become operations this process is called automatization. The reverse process of de-automatization is common in instruction, where learners must often question and analyze implicit assumptions or automated procedures if they turn out to be unproductive.

\section{THE ACTIVITY: KEPLER'S LAWS}

Two three-member groups of 15 year-old high school students from Slovenia were asked to use the given equipment, an IWB with Algodoo software, to "investigate the movement of smaller objects near a massive central object". The instructions were very short (as quoted) and open-ended, since the students were already familiar with the software and have already used it for open-ended inquiry in other topics. Students could create smaller objects by drawing them on the board and manipulate them by dragging them and throwing them around on the IWB. Each group spent 60 minutes working on the IWB. We have videorecorded and transcribed the work of both groups. In the following analysis we have applied the categories of activity theory to get an insight into the processes constituting the observed activity, the setting and conditions in which it took place.

Tools: The IWB is considered to be the tool in the observed activity, while the virtual bodies being sent into orbit were the objects upon which the subjects acted. In the school where the study took place, the IWBs are being used on a regular basis, but they are mostly used as traditional boards and projection screens. We used the IWB coupled with Algodoo software as a tool because the combination of the two offered possibilities for experimentation with, and different representations of, the content. Prior to conducting the experiment with the students, we carefully studied and tested relevant software parameters, such as the size of the Sun and the strength of its attraction, to provide the participants with optimal conditions for exploration. A week prior to the recorded sessions we showed Algodoo software to the 
participants during a brief 10 -minute presentation. We demonstrated to them the basics of Algodoo use on the IWB and encouraged them to download the software to their personal computers and get acquainted with it prior to the activity session. All the participants selfreported to have tested the software on their own computers but were unfamiliar with using Algodoo to explore orbital motion. The participants were familiar with the basics of the software and the touch-screenlike use of IWB. We observed that all the students could manipulate the IWB with relative ease. They appeared to be well acquainted with the screen-gesture "philosophy" and quickly learned to use the quick access gestures of the Algodoo software and other widely present functions, such as "undo".

Rules: One of the researches was present in the room during the experiment. He gave the above-quoted instructions to the participants at the beginning and remained in the back of the room for the rest of the time. The researcher only had to intervene 2 times to prevent students from straying off-topic, once with each group and for the same reason; the students started attaching rocket engines to created objects, which would greatly complicate the activity, so they were asked to first deal with motion without the attached rocket engines.

Subjects - the group micro-community: The groups consisted of three 15 year old participants (three boys in group I and two boys and one girl in group II). They volunteered in class to be a part of a study on the use of IWB in physics. In the school where the study was done, one class per year is the so-called mathematical class. It usually consists of students especially interested in mathematics and natural sciences and has 5 instead of 4 lessons (for a regular class) of math per week. Group I were students from a regular class and were well performing in physics. Group II were from a mathematical class - physics enthusiasts, but not the very best in class, as their teacher put it. The previous knowledge of the group I was basic Newtonian mechanics (no linear momentum, no angular momentum). Group II was much more advanced (two of the members already knew Kepler's laws. During the session they also discussed energy conservation in orbital motion, but did not refer to angular or linear momentum). As it turned out, the subjects "selfregulated" the advancement of activities and so group II ended up exploring more advanced issues than group I, despite the same initial instructions. As we have studied small groups of students, the AT categories of subject and community have been merged for the purpose of this analysis. In the following paragraphs we further describe the emergent group activity.

Division of labor: We did not want to guide students here as observing the emerging dynamics of the group was one of the goals of our study. However, occasional interventions by the researcher have influenced the group dynamics, for example, by encouraging students to express their thoughts or to follow through with their ideas. Occasionally, the researcher would ask a question that would steer the discussion (for an example, see the transcript excerpt provided in the Findings section). The researcher was also engaged in the activity by helping students manage the use of the software when they needed help. Otherwise, we observed that the participants naturally divided the activity, constituent actions and underlying operations among group participants with little input from the researcher. In both sessions, the interactions surrounding the IWB activity, actions or even lowerlevel operations were mostly happening between two students standing close to the IWB, while the third student was often in the role of an observer. The roles shifted during the activity, but there was a clear tendency for one of the participants to be the observer most often. However, the "observer" in both sessions came up with relevant ideas that could be tested by the "performers".

Objects: motives - goals - sub-goals: The objects of the activity under study were the virtual artifacts that were being manipulated using the IWB as the tool. The main goal (the motive in AT terms) of the activity was qualitative exploration of the orbital motion of planets. We planned that students would do quantitative investigations only if they expressed interest and if time permitted. With this in mind, the researcher present in the classroom would only occasionally steer the activity.

Both groups very quickly started sending planets into orbit by either throwing them with the grab tool or sending them into orbit using the built-in option of the software, which led to productive discussions and inquiries. When the students started to refer to certain patterns, the researcher encouraged them to further explore, describe and test those patterns. The sub-goals on the action level were mostly proposed by students (for example, throwing a planet in a highly eccentric orbit to see if its speed really changes depending on its distance from the Sun).

Although students became familiar with the software's intuitive interface and quickly automatized the control gestures, most of the interventions and subgoal setting by the researcher were done at the level of operations, helping the students manage technical difficulties in controlling the software. This usually meant de-automatization of certain operations by setting concrete low-level operational goals. For example, a student would try to grab the planet without clicking on it first, resulting in failure to grab it. Therefore, the researcher would instruct the student to click on the planet before grabbing and throwing it. 


\section{FINDINGS}

The above-presented analysis provides us with answers to the research questions regarding the observed orbital-motion activity.

What will the learners do if they are given full control of the IWB? Students quickly engaged in discussions that accompanied the manipulation of the software on the IWB. Both groups have observed and articulated qualitative patterns of orbital motion described by Kepler's laws. Group II went further and investigated areal velocities of planets quantitatively.

Will the designed activity encourage social interactions and collaborative learning? The artifacts on the board, often a product of collaboration between the participants, were also used as references in discussions, collaborative sense-making and hypotheses testing. The following excerpt from the transcription of group I activity shows a collaborative formulation of a prediction of what will happen if the orbiting planets are suddenly exposed to air drag. The discussion started with an observation that the elliptic orbits of planets otherwise remained stable over time:

Amy: So there is no resistance, because they always follow the same path... Air for example.

Researcher: What do you think would happen if there was resistance?

Amy: Well, the path would probably change somewhat and also the speed (points to the existing trace)... Well, I think it would eventually stop.

Mathew: Yes, it would fall down. If we completely stopped it, it would fall directly. Ted: (Nods)

Mathew: But if we added air drag just now...

Ted: It would get smaller (Gestures a spiral-like shape with his hand, from the existing ellipse and inwards.)

Mathew: The ellipse would slowly get smaller.

Amy: It would slowly get closer (Points towards the Sun with a spiral-like gesture.)

To what extent will the students be kinesthetically engaged? Student familiarity and everyday experience with touch-screen technology seemed to have been of great help when students engaged in the manipulation of on-screen artifacts (grabbing and throwing planets around, attaching tracers to planets, playing and stopping the simulation, etc.). We have counted 435 instances of such software manipulation actions in both sessions (approx. four per minute on average). As can also be seen in the above excerpt, students engaged kinesthetically not only when using the IWB, but also used gesticulation as a representation in discussion (pointing out distances, gesticulating planet movement, etc.). This was observed with all of the six participating students. In both sessions, there were 106 instances of a student using such a gesticulative representation. Content-related learner gesticulation during instruction has been shown to benefit knowledge retention [11].

\section{DISCUSSION}

Based on the findings we can speculate that activities where students interact with an IWB as a touch-screen, might have potential benefits compared to the more traditional uses of IWBs as a writing tool or a projector due to the benefits of touch-screen-like activities. These include student kinesthetic engagement with the on-screen content (touch-screen manipulation and use of gesticulative representations) as well as multiple opportunities for creative graphical input. In our orbital motion activity, the touch-screen affordances of the IWB supported student independent engagement in discovering patterns, collaborative sense-making and setting and testing hypotheses. We suggest such activities are used to complement more established uses of the IWB. However, using the IWB as the only touch-screen in large-enrolment courses has certain limitations that need to be addressed by future research. Researchers may also consider investigating such activities on smaller devices, such as tablets, which allow multiple small groups of students to work simultaneously. Finally, encouraging and teaching the teachers to use the IWB in new ways also remains a challenge for the future. The teacher's role in guiding student inquiry is especially important when using multipurpose software like Algodoo, which in-itself contains few explicit restrictions to the creativity of the users. Therefore, appropriate teacher training must focus not only on the technology, but also pedagogy, if the IWB's teaching potential is to be fully exploited.

\section{REFERENCES}

[1] K. Murcia and R. Sheffield, Australas. J. Educ. Technol. 26, 417 (2010).

[2] H. Smith, S. Higgins, K. Wall, and J. Miller, J. Comput. Assist. Learn. 21, 91 (2005).

[3] P. Mishra and M.J. Koehler, Teach. Coll. Rec. 108, 1017 (2006).

[4] M. Wilson, Psychon. Bull. Rev. 9, 625 (2002).

[5] J.M. Iverson and S. Goldin-Meadow, Nature 396, 228 (1998)

[6] L. Vygotsky, Mind in Society: The Development of Higher Psychological Processes (Harvard University Press, 1978).

[7] G. Stahl, T. Koschmann, and D. Suthers, in Cambridge Handb. Learn. Sci., edited by R.K. Sawyer (Cambridge University Press, 2006), pp. 409-426.

[8] F. Erickson, in Handb. Res. Teach., edited by M.C. Wittrock (Macmillan, New York, 1986), pp. 119-161.

[9] V. Kaptelinin and B.A. Nardi, Acting with Technology: Activity theory and interaction design (MIT Press, 2009).

[10] M. Cole and Y. Engeström, in Distrib. Cogn.: Psychol. and Educ. Considerations, edited by G. Salomon (Cambridge University Press, 1993), pp. 1-46.

[11] S. Wagner Cook, Z. Mitchell, and S. Goldin-Meadow, Cognition 106, 1047 (2008). 